\title{
Theory of the firm: bargaining and competitive equilibrium
}

Citation for published version (APA):

Britz, V., Herings, P. J. J., \& Predtetchinski, A. (2010). Theory of the firm: bargaining and competitive equilibrium. METEOR, Maastricht University School of Business and Economics. METEOR Research Memorandum No. 057 https://doi.org/10.26481/umamet.2010057

Document status and date:

Published: 01/01/2010

DOI:

10.26481/umamet.2010057

Document Version:

Publisher's PDF, also known as Version of record

\section{Please check the document version of this publication:}

- A submitted manuscript is the version of the article upon submission and before peer-review. There can be important differences between the submitted version and the official published version of record.

People interested in the research are advised to contact the author for the final version of the publication, or visit the DOI to the publisher's website.

- The final author version and the galley proof are versions of the publication after peer review.

- The final published version features the final layout of the paper including the volume, issue and page numbers.

Link to publication

\footnotetext{
General rights rights.

- You may freely distribute the URL identifying the publication in the public portal. please follow below link for the End User Agreement:

www.umlib.nl/taverne-license

Take down policy

If you believe that this document breaches copyright please contact us at:

repository@maastrichtuniversity.nl

providing details and we will investigate your claim.
}

Copyright and moral rights for the publications made accessible in the public portal are retained by the authors and/or other copyright owners and it is a condition of accessing publications that users recognise and abide by the legal requirements associated with these

- Users may download and print one copy of any publication from the public portal for the purpose of private study or research.

- You may not further distribute the material or use it for any profit-making activity or commercial gain

If the publication is distributed under the terms of Article $25 \mathrm{fa}$ of the Dutch Copyright Act, indicated by the "Taverne" license above, 


\section{Maastricht University}

Volker Britz, J ean-J acques Herings, Arkadi Predtetchinski

Theory of the Firm: Bargaining and Competitive Equilibrium

RM/ 10/057

\section{METEOR}

Maastricht University School of Business and Economics

Maastricht Research School of Economics

of Technology and Organization

P.O. Box 616

NL - 6200 MD Maastricht

The Netherlands 


\title{
Theory of the Firm: Bargaining and Competitive Equilibrium $^{1}$
}

\author{
Volker Britz $^{2} \quad$ P. Jean-Jacques Herings ${ }^{3} \quad$ Arkadi Predtetchinski ${ }^{4}$
}

November 15,2010

${ }^{1}$ The authors would like to thank Jacques Drèze for helpful comments and suggestions.

${ }^{2}$ V. Britz (v.britz@maastrichtuniversity.nl), Department of Economics, Maastricht University. This author would like to thank the Netherlands Organisation for Scientific Research (NWO) for financial support.

${ }^{3}$ P.J.J. Herings (p.herings@maastrichtuniversity.nl). Department of Economics, Maastricht University. This author would like to thank the Netherlands Organisation for Scientific Research (NWO) for financial support.

${ }^{4}$ A. Predtetchinski (a.predtetchinski@maastrichtuniversity.nl), Department of Economics, Maastricht University. This author would like to thank the Netherlands Organisation for Scientific Research (NWO) for financial support. 


\begin{abstract}
Suppose that a firm has several owners and that the future is uncertain in the sense that one out of many different states of nature will realize tomorrow. An owner's time preference and risk attitude will determine the importance he places on payoffs in the different states. It is a well-known problem in the literature that under incomplete asset markets, a conflict about the firm's objective function tends to arise among its owners. In this paper, we take a new approach to this problem, which is based on non-cooperative bargaining. The owners of the firm play a bargaining game in order to choose the firm's production plan and a scheme of transfers which are payable before the uncertainty about the future state of nature is resolved. We analyze the resulting firm decision in the limit of subgame-perfect equilibria in stationary strategies. Given the distribution of bargaining power, we obtain a unique prediction for a production plan and a transfer scheme. When markets are complete, the production plan chosen corresponds to the profit-maximizing production plan as in the Arrow-Debreu model. Contrary to that model, owners typically do use transfers to redistribute profits. When markets are incomplete, the production plan chosen is almost always different from the standard notion of competitive equilibrium and again owners use transfers to redistribute profits. Nevertheless, our results do support the Drèze criterion as the appropriate objective function of the firm.
\end{abstract}

KEYwORDs: Strategic bargaining, Nash bargaining solution, incomplete markets, stock market equilibrium, objective function of the firm, profit-maximization.

JEL CODES: C78, D52. 


\section{Introduction}

Suppose that a firm has several owners and that the future is uncertain in the sense that one out of many different states of nature will realize tomorrow. An owner's time preference and risk attitude will determine the importance he places on payoffs in the different states. It is a well-known problem in the literature that under incomplete asset markets, a conflict about the firm's objective function tends to arise among its owners.

We take a non-cooperative bargaining approach to this conflict, which is new to the literature. We present a model in which the internal decision making of the firm is formalized explicitly as a strategic bargaining game. In contrast to standard theory, we thus consider the firm as a coalition of owners who use strategic power in order to influence the firm's production decision and thereby maximize their own payoffs.

The standard approach in the existing literature on incomplete markets with production originates from contributions by Diamond (1967), Drèze (1974), and Grossman and Hart (1979). Diamond (1967) formulates the notion of constrained Pareto optimality, an optimality notion that takes the incompleteness of asset markets into account. Drèze (1974) shows that necessary first-order conditions for constrained Pareto optimality require that the firm should choose a production plan which is optimal when evaluated against a weighted average of the shareholders' utility gradients, where the weights are given by the ownership shares. This objective function for the firm is usually referred to as the Drèze criterion.

Standard notions of constrained Pareto optimality allow for arbitrary redistributions in the initial period, and so does Drèze (1974), pp. 141-142:

"The definition does not place any restrictions on the allocation among consumers of the adjustments in current consumption required to offset the adjustment in the input level $a^{j}-\bar{a}^{j}$. Alternatively stated, the definition is consistent with arbitrary transfers of initial resources among consumers."

Arbitrary redistributions in the initial period leads to indeterminateness of equilibrium. With $H$ shareholders, one would in general expect a multiplicity of degree $H-1$.

Grossman and Hart (1979) use the Drèze criterion as the objective function for the firm, but with final ownership shares replaced by initial ones. Contrary to Drèze (1974), they also require that at equilibrium no transfers are made in the initial period. The advantage of this approach is that it leads to determinateness of equilibrium, its downside is inconsistency. Indeed, initial period transfers are used in Grossman and Hart (1979) in their definition of constrained Pareto optimality, which motivates the decision criterion for the firm. At 
the same time, the equilibrium decision of the firm is imposed to involve no transfers itself. Absence of initial-period transfers has become the standard concept of equilibrium, called stock market equilibrium, see for instance the treatment in the authoritative book of Magill and Quinzii (1996) and in the paper by Dierker, Dierker, and Grodal (2002) on the constrained inefficiency of stock market equilibria.

Recognizing that the Drèze criterion is normative in nature, some authors have tried to link it to outcomes of majority voting. A typical approach in this stream of literature is to ask: If a certain production plan was given as a default option, would there exist an alternative plan preferred by more than at least a certain (super-)majority of the shareholders? If no winning alternative can be found, the default plan is considered "stable." Tvede and Crès (2005) discuss the relationship between the Drèze criterion and such a voting approach and find conditions under which both lead to compatible predictions. Another voting analysis is given by DeMarzo (1993) who emphasizes the importance of the largest shareholder. Kelsey and Milne (1996) give a proof for equilibrium existence in a more general model which emphasizes externalities between firms and shareholders. However, both stock market equilibria and the approaches related to majority voting seem to suffer from a common problem. Both approaches ask only which production plans are stable to being replaced by other plans through a certain mechanism. However, there is no explanation why a particular plan would serve as a default setting or how any one particular plan is to be chosen in case there are several plans satisfying the criterion used.

Few papers have taken a truly positive approach to decision making within the firm, as is the case in this paper.

In our setup, there is a single firm which exists in an environment of competitive but potentially incomplete markets. We take the ownership structure of the firm as exogenously fixed to focus attention on the decision making within the firm, rather than on the role of expectations regarding future stock price as a consequence of current production decisions and the identity of future shareholders as in Bonnisseau and Lachiri (2004) and Drèze, Lachiri, and Minelli (2009). When applied to our setting without stock markets, we refer to the stock market equilibrium concept as competitive equilibrium.

The firm will be active in two time periods. A production plan and a transfer scheme have to be chosen knowing the state of the world in the first period, but under uncertainty about the state of the world in the second period. There are assets which the owners can use to shift consumption across time periods and states. Owners are price-takers in the asset markets.

We address the issue raised in Magill and Quinzii (1996), who write on p. 364 when referring to the concept of partnership equilibrium: 
"A weakness of this concept of equilibrium is that it does not provide a welldefined bargaining process by which partners could come up with such an agreement."

In this paper, we propose such a bargaining process. We have the owners of the firm bargain in order to determine a production plan for the firm. In each round of bargaining, an owner is chosen to make a proposal. This owner offers a production plan as well as a transfer scheme of side-payments in terms of first-period consumption. If the owners unanimously agree, the proposal is implemented. Otherwise, the negotiation breaks down with some probability, in which case no production takes place and no transfers are made. With the complementary continuation probability, bargaining continues to another bargaining round. In the case of perpetual disagreement, the firm will not produce, and no transfers will be made. Through the bargaining process, the owners of the firm collectively decide on a production plan and a transfer scheme. Moreover, as individuals they may invest in the asset markets to determine their amounts of consumption at each state of the world. We refer to our notion of equilibrium as bargaining equilibrium.

Our main results are the following. We show that the bargaining equilibrium corresponds to a weighted Nash Bargaining Solution; a unique prediction for a production plan as well as a system of transfer payments in terms of good 0 is derived. In the special case where markets are complete, the bargaining equilibrium selects the profit-maximizing production plan and is therefore in line with the predictions of the Arrow-Debreu model as far as the firm's production decision is concerned. However, contrary to the Arrow-Debreu model, owners make use of their bargaining power to redistribute the profits among themselves via the transfer scheme. Hence, owners obtain payoffs which are generically different from those in standard economic theory, even if markets are complete.

In the case of incomplete markets, we find that the production plan which the firm adopts under the bargaining equilibrium is almost always different from the competitive equilibrium one. Moreover, non-zero transfers are made in general. At the same time, bargaining equilibria are constrained Pareto optimal, and are therefore consistent with the use of the Drèze criterion. However, since contrary to competitive equilibrium transfers are made, the shareholders' utility gradients are not the same as the competitive equilibrium ones, explaining why the chosen production plan does typically not coincide with the one corresponding to a competitive equilibrium. The bargaining equilibrium satisfies the requirements of an equilibrium as defined in Drèze (1974). Like the competitive equilibrium, the bargaining equilibrium can therefore be viewed as selecting a particular Drèze equilibrium. 
In Section 2, we present the model and the most important assumptions formally. In Section 3, we show how bargaining on the production plan and a transfer scheme can be reformulated as bargaining on the implied payoffs. In Section 4, we characterize the relevant bargaining outcome. In Section 5, this bargaining outcome is interpreted in light of the existing literature and, in particular, compared to the Drèze criterion. Section 6 concludes.

\section{Model Description}

We study a firm with several owners in a setting with incomplete markets. Each owner $i=1, \ldots, I$ holds a share $\theta^{i}>0$ of the firm so that $\sum_{i=1}^{I} \theta^{i}=1$. The set of owners $\{1, \ldots, I\}$ will be denoted by $\mathcal{I}$.

The firm carries out some productive activity which extends over two periods. In the first period, the state of the world is known to be $s=0$. In the second period, any one of the states of the world $s=1, \ldots, S$ may be realized; we write $\mathcal{S}=\{0,1, \ldots, S\}$. A particular productive activity of the firm is described by a production plan $y \in \mathbb{R}^{S+1}$. If $y_{s}<0$ for some $s \in \mathcal{S}$, then each owner $i$ has to provide the firm with an input of $\left|\theta^{i} y_{s}\right|$ in state $s$. Similarly, if $y_{s}>0$, then this output will be distributed to the owners in the proportion in which they own the firm. The set of all production plans which are feasible for the firm is called its production set and is denoted by $Y$.

Each owner $i$ has initial endowments $\omega^{i} \in \mathbb{R}_{++}^{S+1}$, which can be used to finance the provision of inputs. Consumption in state $s=0$ can be transferred across owners and assets can be used to shift consumption across states. Transfers and assets will be introduced in detail in the sequel.

A production plan can be chosen only by unanimous consent of the owners. In order to reach agreement, the following bargaining procedure is used. Bargaining takes place in a, potentially infinite, number of rounds $r=0,1, \ldots$ In the beginning of any round $r$, a draw from a given probability distribution $\mu \in \Delta^{I}$, where $\Delta^{I}$ is the set of strictly positive vectors in the unit simplex in $\mathbb{R}^{I}$, determines the proposer in round $r$. This proposer then makes an offer $(y, t) \in Y \times T$, where $T=\left\{t \in \mathbb{R}^{I} \mid \sum_{i \in \mathcal{I}} t^{i} \leq 0\right\}$. Here $t^{i}$ denotes the transfer owner $i$ receives, and which is made in terms of consumption in state 0 . The owners then either accept or reject the offer in some given order. It is assumed that an owner cannot accept a proposal which leads to his insolvency irrespective of his choice of an asset portfolio. This assumption will be stated more formally later on.

If unanimous agreement on the production plan and transfer scheme is reached, the bar- 
gaining stage ends and the chosen production plan and transfer scheme are implemented. If, however, an owner rejects a proposal, bargaining moves to round $r+1$ with probability $\delta$. With probability $1-\delta$, a breakdown of bargaining occurs. In that case, we assume that no production will take place and no transfers are made. Each individual owner is merely left to choose his asset portfolio. Likewise, perpetual disagreement means that no production takes place. One interpretation of the breakdown probability is that the investment opportunities implicit in the production set may slip away if one waits too long to exploit them.

Once bargaining has led to an agreement (or broken down), the sequel of the model does not incorporate any strategic interaction anymore. Each owner individually decides on an asset portfolio. The owner can purchase assets $j=1, \ldots, J$ at exogenously given prices $q_{1}, \ldots, q_{J}$. Owners act as price takers in the asset markets. In state $s=1, \ldots, S$, each unit of asset $j$ will give a payoff of $a_{s}^{j}$. We summarize the asset structure in the $(S \times J)$-matrix $A$, which we assume to be of full column rank. Redundant assets are ignored without loss of generality. It will sometimes be convenient to use the notation

$$
W=\left(\begin{array}{c}
-q \\
A
\end{array}\right) .
$$

Assets are perfectly divisible and may be sold short. We write $z_{j}^{i}$ for owner $i$ 's holdings of asset $j$.

Economic activity in state 0 therefore consists of the implementation of the agreed production plan and transfers, the choice of asset portfolios, and consumption. Next, one state of nature $s \in\{1, \ldots, S\}$ realizes, and contingent on $s$ the assets pay off, the firm realizes its output, and the owners consume.

Owner $i$ has preferences over consumption plans $x^{i} \in \mathbb{R}_{++}^{S+1}$, which are represented by a von Neumann-Morgenstern utility function $u^{i}: \mathbb{R}_{++}^{S+1} \rightarrow \mathbb{R}$. Given the bargaining outcome $(y, t) \in Y \times T$, owner $i$ solves the following maximization problem:

$$
\begin{aligned}
\max _{x^{i} \in \mathbb{R}_{++}^{S+1}, z^{i} \in \mathbb{R}^{J}} u^{i}\left(x^{i}\right) \text { subject to } x_{0}^{i} & =\omega_{0}^{i}+\theta^{i} y_{0}+t^{i}-q z^{i}, \\
x_{s}^{i} & =\omega_{s}^{i}+\theta^{i} y_{s}+A_{s} z^{i}, s=1, \ldots, S .
\end{aligned}
$$

Let $e(0)$ denote the $(S+1)$-dimensional column vector $(1,0, \ldots, 0)^{\top}$. For $i \in \mathcal{I}$, we define

$$
B^{i}=\left\{(y, t) \in Y \times T \mid \exists z^{i} \in \mathbb{R}^{J}, \omega^{i}+\theta^{i} y+e(0) t^{i}+W z^{i} \gg 0\right\},
$$

and $B=\cap_{i \in \mathcal{I}} B^{i}$. The set $B$ contains all bargaining outcomes which allow each owner to remain solvent by an appropriate portfolio choice. Solvency in this context means a strictly 
positive consumption in each state. Owner $i$ can only accept a proposal if it belongs to $B^{i}$. Since $\omega^{i} \gg 0$ for all $i \in \mathcal{I}$, it holds that $(0,0) \in B$ and thus $B$ is non-empty.

The results in this paper are derived under a number of assumptions on the utility functions, the production possibility set, and the asset structure. These assumptions are now introduced.

\section{Assumption 2.1 (Production Set)}

1. $Y$ is closed, strictly convex, and bounded from above.

2. $Y \supset \mathbb{R}_{-}^{S+1}$ : Output can be freely disposed of and inaction is possible.

3. $Y \cap \mathbb{R}_{+}^{S+1} \subset\{0\}:$ One cannot produce a positive output without inputs.

4. $\partial Y$ is a $C^{2}$ manifold with nonzero Gaussian curvature.

\section{Assumption 2.2 (Utility functions)}

For all $i \in \mathcal{I}$ we assume the following.

1. $u^{i}$ is twice continuously differentiable on $\mathbb{R}_{++}^{S+1}$.

2. $u^{i}$ is differentiably strictly concave on $\mathbb{R}_{++}^{S+1}$, i.e. the Hessian matrix of $u^{i}$ is negative definite on $\mathbb{R}_{++}^{S+1}$.

3. For any $s=0,1, \ldots, S$, and any $x_{-s}^{i} \in \mathbb{R}_{++}^{S}$ it holds that $u^{i}\left(x_{s}^{i}, x_{-s}^{i}\right)$ goes to negative infinity in the limit as $x_{s}^{i}$ approaches zero.

We define the set of normalized state prices $\Pi$ by

$$
\Pi=\left\{\pi \in \mathbb{R}_{++}^{S} \mid(1, \pi) W=0\right\} .
$$

\section{Assumption 2.3 (Asset Structure)}

1. The matrix $A$ has full column-rank.

2. The set $\Pi$ is non-empty.

3. There is $\pi \in \Pi$ such that $\pi$ is not normal to $Y$ at $y=0$.

Assumption 2.3.2 would be satisfied in general equilibrium, but has to be imposed here since we conduct our analysis in a partial equilibrium context. Assumption 2.3.3 rules out the (uninteresting) special case in which markets are complete and the unique profitmaximizing production plan is $y=0$.

Asset markets are said to be complete if $\Pi$ is single-valued. If $\Pi$ is not single-valued, markets are called incomplete. We also note that $\Pi$ is a convex set. 


\section{Reduced Form Bargaining Game}

When the owners bargain about a production plan and a transfer scheme, they implicitly bargain about the associated payoffs. In this section, we will analyze the bargaining problem in payoff space. In order to motivate this approach, we will show that, given efficiency and individual rationality, there is a one-to-one correspondence between both problems. In other words, any Pareto-efficient and individually rational outcome of the bargaining problem in the payoff space is supported by a unique combination of a production plan, a transfer scheme, and asset portfolios for each owner. This result is driven by the assumptions of strict convexity of the production set and strict concavity of the utility functions.

Consider some $(y, t) \in B$. By definition of $B$, there must be some $z \in \mathbb{R}^{I J}$ such that the vector $x^{i}=\omega^{i}+\theta^{i} y+e(0) t^{i}+W z^{i}$ is strictly positive for all $i \in \mathcal{I}$. Using Assumption 2.3 , we see that the set of portfolio choices for owner $i$ which lead to a utility of at least $u^{i}\left(x^{i}\right)$ is compact. Hence, the indirect utility $\bar{u}^{i}(y, t)$ is well-defined. We write $\bar{u}(y, t)$ for the profile of utilities $\left(\bar{u}^{1}(y, t), \ldots, \bar{u}^{I}(y, t)\right)$. It holds that the optimal consumption bundle, denoted by $\xi^{i}(y, t)$, is unique. Suppose to the contrary that there are two distinct feasible consumption bundles $x^{i}$ and $\bar{x}^{i}$ leading to utility $\bar{u}^{i}(y, t)$ Since any convex combination of $x^{i}$ and $\bar{x}^{i}$ is feasible and gives strictly higher utility than $\bar{u}^{i}(y, t)$, we have a contradiction. The uniqueness of $\xi^{i}(y, t)$ combined with the full column-rank of $A$ implies that the optimal portfolio choice is unique. The set $V$ of feasible payoffs for the owners is given by $V=\bar{u}(B)$, a subset of $\mathbb{R}^{I}$.

We denote by $V^{+}$the individually rational part of $V$, and by $\partial V$ and $\partial V^{+}$the weak Pareto boundaries of the sets $V$ and $V^{+}$. Individually rational payoffs are here defined as the payoffs that exceed the payoffs $v^{0}=\bar{u}(0,0)$ owners could achieve without relying on firm production and transfers, but by making use of trades in the asset market. We define

$$
\begin{aligned}
V^{+} & =\left\{v \in V \mid v \geq v^{0}\right\}, \\
\partial V & =\left\{v \in V \mid \nexists v^{\prime} \in V, v^{\prime} \gg v\right\}, \\
\partial V^{+} & =V^{+} \cap \partial V .
\end{aligned}
$$

The next five lemmas state that the set $V^{+}$satisfies a number of desirable properties. In particular, we demonstrate that $V^{+}$is compact and convex, that $V$ is comprehensive from below, that all points in $\partial V^{+}$are strongly Pareto efficient, and that there is a unique vector in the normal to $V$ at any point in $\partial V^{+}$.

Lemma 3.1 The set $V$ is comprehensive from below. 
Proof: Take $\bar{v} \in V$. We want to show that any $v \in \mathbb{R}^{I}$ such that $v \leq \bar{v}$ belongs to $V$. Let $(\bar{x}, \bar{y}, \bar{t}, \bar{z}) \in \mathbb{R}_{++}^{(S+1) I} \times B \times \mathbb{R}^{J I}$ be such that $\bar{v}=\bar{u}(\bar{y}, \bar{t})$ and let $(\bar{x}, \bar{z})$ correspond to optimal consumption choices and portfolio plans given $(\bar{y}, \bar{t})$. Consider a particular $i \in \mathcal{I}$, and define a set $L^{i}$ as follows.

$$
L^{i}=\left\{x^{i} \in \mathbb{R}_{+}^{S+1} \mid \exists z^{i} \in \mathbb{R}^{J}, x^{i}=\omega^{i}+\theta^{i} \bar{y}+e(0) \bar{t}^{i}+W z^{i}\right\} .
$$

The set $L^{i}$ is non-empty, closed, and bounded. Thus, there exists $\hat{x}^{i} \in L^{i}$ such that $\hat{x}_{0}^{i} \geq x_{0}^{i}$ for all $x^{i} \in L^{i}$. Let $\hat{z}^{i}$ be the asset portfolio associated with $\hat{x}^{i}$.

For some $\kappa \in(0,1)$, define $\tilde{x}^{i}=\kappa \bar{x}^{i}+(1-\kappa)\left(0, \hat{x}_{1}^{i}, \ldots, \hat{x}_{S}^{i}\right)^{\top}$. The consumption plan $\tilde{x}^{i}$ results from the production plan $\bar{y}$, the transfer $\tilde{t}^{i}:=\bar{t}^{i}-(1-\kappa) \hat{x}_{0}^{i}$, and the asset portfolio $\tilde{z}^{i}=\kappa \bar{z}^{i}+(1-\kappa) \hat{z}^{i}$.

Since $\kappa$ is strictly positive by construction, we have that $\tilde{x}^{i} \in \mathbb{R}_{++}^{S+1}$, and therefore $(\bar{y}, \tilde{t}) \in B^{i}$.

By construction of $\hat{x}^{i}$ and $\tilde{t}^{i}$, it holds that $\xi_{0}^{i}(\bar{y}, \tilde{t}) \leq \hat{x}_{0}^{i}-\bar{t}^{i}+\tilde{t}^{i}=\kappa \hat{x}_{0}^{i}$.

We can use a direct argument to show that $\xi^{i}$ is continuous in $(y, t) \in B^{i}$, and we have assumed that the direct utility function $u^{i}$ is continuous on $\mathbb{R}_{++}^{S+1}$. Thus, the indirect utility function $\bar{u}^{i}=u^{i} \circ \xi^{i}$ is continuous and reaches any value in the interval $\left[\bar{u}^{i}(\bar{y}, \tilde{t}), \bar{u}^{i}(\bar{y}, \bar{t})\right]$. The statement follows from passing to the limit as $\kappa \downarrow 0$, in which case $\xi_{0}^{i}(\bar{y}, \tilde{t})$ becomes arbitrarily small and Assumption 2.2.3 implies that $\bar{u}^{i}(\bar{y}, \tilde{t})$ becomes arbitrarily negative. Finally, notice that our construction for owner $i$ only involves the transfer $t^{i}$, so that we can deal with the functions $\bar{u}^{1}, \ldots, \bar{u}^{I}$ independently.

We have defined $\partial V^{+}$as the weak Pareto-boundary of $V^{+}$. We will show next that it coincides with the strong Pareto-boundary. That is, $\bar{v}$ belongs to $\partial V^{+}$if and only if there is no $v \in V$ such that $v \geq \bar{v}$, with strict inequality in at least one component.

Lemma 3.2 All points in $\partial V^{+}$are strongly Pareto-efficient.

Proof: Consider some $v \in \partial V^{+}$. Let $(x, y, t, z) \in \mathbb{R}_{++}^{(S+1) I} \times B \times \mathbb{R}^{J I}$ be such that $v=\bar{u}(y, t)$ and let $(x, z)$ correspond to an optimal consumption choice and portfolio plan given $(y, t)$. Suppose that there is $\bar{v} \in V$ such that $\bar{v}^{i^{\prime}}>v^{i^{\prime}}$ for some $i^{\prime} \in \mathcal{I}$ and $\bar{v}^{i} \geq v^{i}$ for all $i \in \mathcal{I} \backslash\left\{i^{\prime}\right\}$. Let $(\bar{x}, \bar{y}, \bar{t}, \bar{z}) \in \mathbb{R}_{++}^{(S+1) I} \times B \times \mathbb{R}^{J I}$ be such that $\bar{v}=\bar{u}(\bar{y}, \bar{t})$ and let $(\bar{x}, \bar{z})$ correspond to an optimal consumption choice and portfolio plan given $(\bar{y}, \bar{t})$. For $\varepsilon \in\left(0, \bar{x}_{0}^{i^{\prime}}\right)$, we construct the consumption plan $\tilde{x}^{i^{\prime}}=\bar{x}^{i^{\prime}}-\varepsilon e(0)$ and $\tilde{x}^{i}=\bar{x}^{i}+(\varepsilon /(I-$ 1))e(0) for $i \in \mathcal{I} \backslash\left\{i^{\prime}\right\}$. Since $u^{i^{\prime}}$ is continuous, $u^{i^{\prime}}\left(\tilde{x}^{i^{\prime}}\right)>u^{i^{\prime}}\left(x^{i^{\prime}}\right)$ for $\varepsilon$ sufficiently small. Assumption 2.2.2 implies that $u^{i}\left(\tilde{x}^{i}\right)>u^{i}\left(x^{i}\right)$ for all $i \in \mathcal{I} \backslash\left\{i^{\prime}\right\}$. We define the transfer 
scheme $\tilde{t} \in T$ by $\tilde{t}^{i^{\prime}}=\bar{t}^{i^{\prime}}-\varepsilon e(0)$ and $\tilde{t}^{i}=\bar{t}^{i}+(\varepsilon /(I-1)) e(0)$ for $i \in \mathcal{I} \backslash\left\{i^{\prime}\right\}$. Then $\bar{u}(\bar{y}, \tilde{t}) \geq\left(u^{1}\left(\tilde{x}^{1}\right), \ldots, u^{I}\left(\tilde{x}^{I}\right)\right) \gg v$, a contradiction to the weak Pareto efficiency of $v$.

Lemma 3.3 The set $V^{+}$is compact.

\section{Proof:}

We define the set $X$ as follows,

$$
X=\left\{x \in \mathbb{R}_{+}^{(S+1) I} \mid \exists(y, t, z) \in B \times \mathbb{R}^{J I}, \forall i \in \mathcal{I}, x^{i}=\omega^{i}+\theta^{i} y+e(0) t^{i}+W z^{i}\right\} .
$$

We will show first that $X$ is bounded.

Since $X$ is a subset of $\mathbb{R}_{+}^{(S+1) I}$, it is clearly bounded from below. To show it is bounded from above, take any $x \in X$ and compute

$$
\sum_{i \in \mathcal{I}} x^{i}=\sum_{i \in \mathcal{I}} \omega^{i}+y+e(0) \sum_{i \in \mathcal{I}} t^{i}+W \sum_{i \in \mathcal{I}} z^{i} \leq \sum_{i \in \mathcal{I}} \omega^{i}+y+W \sum_{i \in \mathcal{I}} z^{i} .
$$

By Assumption 2.3.2, the set $\Pi$ is non-empty. For $\pi \in \Pi$ it holds that $\pi \in \mathbb{R}_{++}^{S}$ and $(1, \pi) W=0$, so

$$
(1, \pi) \sum_{i \in \mathcal{I}} x^{i} \leq(1, \pi)\left(\sum_{i \in \mathcal{I}} \omega^{i}+y\right) .
$$

It follows that $(1, \pi) \sum_{i \in \mathcal{I}} x^{i}$ is bounded from above since $Y$ is bounded from above by Assumption 2.1.1. Since $x^{i}$ is bounded from below for all $i,(1, \pi) \sum_{i \in \mathcal{I}} x^{i}$ bounded from above implies that $x^{i}$ is bounded from above for all $i \in \mathcal{I}$. We have shown that $X$ is bounded.

The boundedness of $X$ and Assumption 2.2.3 imply that for each $i \in \mathcal{I}$ there is a vector $b^{i} \in \mathbb{R}_{++}^{S+1}$ such that $x \in X$ and $u^{i}\left(x^{i}\right) \geq v^{0 i}$ imply $x^{i} \geq b^{i}$.

Now define the set

$$
X^{*}=X \cap\left\{x \in \mathbb{R}_{+}^{(S+1) I} \mid x^{i} \geq b^{i}, \forall i \in \mathcal{I}\right\} .
$$

Since $X^{*} \subset X$, it is immediate that $X^{*}$ is bounded. We will show next that $X^{*}$ is closed. To this end, we define the sets

$$
\begin{aligned}
\tilde{Y} & =\left\{\left(\theta^{1} y, \ldots, \theta^{I} y\right) \in \mathbb{R}^{(S+1) I} \mid y \in Y\right\}, \\
\tilde{T} & =\left\{\tau \in \mathbb{R}^{(S+1) I} \mid \sum_{i \in \mathcal{I}} \tau_{0}^{i} \leq 0 ; \forall i \in \mathcal{I}, \forall s \neq 0, \tau_{s}^{i}=0\right\}, \\
\tilde{W} & =\prod_{i \in \mathcal{I}} \mathcal{R}(W),
\end{aligned}
$$

where $\mathcal{R}(W)$ denotes the column space of $W$. We can write $X^{*}$ as the intersection of the closed set $\left\{x \in \mathbb{R}_{+}^{(S+1) I} \mid x^{i} \geq b^{i}, \forall i \in \mathcal{I}\right\}$ and the set $\{\omega\}+\tilde{Y}+\tilde{T}+\tilde{W}$. We show the latter set to be closed, thereby proving that $X^{*}$ is closed. 
To show that $\{\omega\}+\tilde{Y}+\tilde{T}+\tilde{W}$ is closed, we show that the asymptotic cones of the terms in the sum are positively semi-independent. Since $\{\omega\}$ is bounded, the asymptotic cone $\mathrm{A}(\{\omega\})=\{0\}$. Since $\tilde{Y}$ is bounded from above, $\mathrm{A}(\tilde{Y})$ is contained in $-\mathbb{R}_{+}^{(S+1) I}$. Since $\tilde{T}$ and $\tilde{W}$ are cones themselves, they coincide with their asymptotic cones.

Let $y, \tau$, and $w$ be elements of $\mathrm{A}(\tilde{Y}), \mathrm{A}(\tilde{T})$, and $\mathrm{A}(\tilde{W})$ summing up to zero. We show that $y, \tau$, and $w$ are zero vectors, thereby proving that the asymptotic cones are positively semi-independent. For $i \in \mathcal{I}$, let $z^{i} \in \mathbb{R}^{J}$ be such that $w^{i}=W z^{i}$. We have that

$$
\begin{array}{ll}
y_{0}^{i}+\tau_{0}^{i}-q z^{i}=0, & i \in \mathcal{I}, \\
y_{-0}^{i}+A z^{i}=0, & i \in \mathcal{I} .
\end{array}
$$

We take a weighted sum of these equalities, with weights equal to $(1, \pi)$ for some $\pi \in \Pi$ and obtain

$$
y_{0}^{i}+\tau_{0}^{i}-q z^{i}+\pi y_{-0}^{i}+q z^{i}=0, \quad i \in \mathcal{I} .
$$

Finally, we take the sum over $i \in \mathcal{I}$ and find

$$
\sum_{i \in \mathcal{I}} y_{0}^{i}+\sum_{i \in \mathcal{I}} \tau_{0}^{i}+\pi \sum_{i \in \mathcal{I}} y_{-0}^{i}=0
$$

Since the vector $\pi$ is strictly positive, $\sum_{i \in \mathcal{I}} \tau_{0}^{i} \leq 0$, and $y^{i} \in-\mathbb{R}_{+}^{S+1}$, we find that $y^{i}=0$ for all $i \in \mathcal{I}$ and $\sum_{i \in \mathcal{I}} \tau_{0}^{i}=0$. For all $i \in \mathcal{I}$, since $0=y_{-0}^{i}=-A z^{i}$, the full column-rank of $A$ implies that $z^{i}=0$, and consequently that $w^{i}=0$. Now it holds that $\tau_{0}^{i}=-y_{0}^{i}-w_{0}^{i}=0$. We have shown that the set $X^{*}$ is closed.

We have assumed the utility functions to be continuous on the strictly positive orthant. By definition, $X^{*} \subset \mathbb{R}_{++}^{(S+1) I}$, and so the utility functions are continuous on $X^{*}$, which we have just shown to be a compact set. Hence, the set $U=\left\{v \in \mathbb{R}^{I} \mid \exists x \in X^{*}, v=\right.$ $\left.\left(u^{1}\left(x^{1}\right), \ldots, u^{n}\left(x^{n}\right)\right)\right\}$ is compact as well. We argue next that $V^{+}$coincides with $U \cap\{v \in$ $\left.\mathbb{R}^{I} \mid v \geq v^{0}\right\}$, so is the intersection of a compact set and a closed set, and therefore compact, proving the result. It follows from the definition of the vectors $b^{1}, \ldots, b^{I}$ that $V^{+} \subset U \cap\left\{v \in \mathbb{R}^{I} \mid v \geq v^{0}\right\}$.

Consider some $v \in U \cap\left\{v \in \mathbb{R}^{I} \mid v \geq v^{0}\right\}$. Let $(x, y, t, z) \in X^{*} \times B \times \mathbb{R}^{J I}$ be such that, for all $i \in \mathcal{I}, x^{i}=\omega^{i}+\theta^{i} y+e(0) t^{i}+W z^{i}$, and $u^{i}\left(x^{i}\right)=v^{i}$. Since $\bar{u}(y, t) \geq v \geq v^{0}$ and since $V$ is comprehensive from below by Lemma 3.1, we have that $v \in V^{+}$.

Lemma 3.4 The set $V^{+}$is convex. 
Proof: Let $v, \bar{v}$ be distinct elements of $V^{+}$, and $\tilde{v}=\alpha v+(1-\alpha) \bar{v}$ for some $\alpha \in(0,1)$.

There are $(y, t),(\bar{y}, \bar{t}) \in B$ such that $\bar{u}(y, t)=v$ and $\bar{u}(\bar{y}, \bar{t})=\bar{v}$. Let $z$ and $\bar{z}$ be such that for all $i \in \mathcal{I}, \xi^{i}(y, t)=\omega^{i}+\theta^{i} y+e(0) t^{i}+W z^{i}$, and $\xi^{i}(\bar{y}, \bar{t})=\omega^{i}+\theta^{i} \bar{y}+e(0) \bar{t}^{i}+W \bar{z}^{i}$.

We define $(\tilde{y}, \tilde{t})=\alpha(y, t)+(1-\alpha)(\bar{y}, \bar{t})$ and, for $i \in \mathcal{I}, \tilde{x}^{i}=\alpha \xi^{i}(y, t)+(1-\alpha) \xi^{i}(\bar{y}, \bar{t})$. Since $B$ is convex, we have that $(\tilde{y}, \tilde{t}) \in B$. Furthermore, since

$$
\tilde{x}^{i}=\omega^{i}+\theta^{i} \tilde{y}+e(0) \tilde{t}^{i}+W\left(\alpha z^{i}+(1-\alpha) \bar{z}^{i}\right),
$$

the consumption plan $\tilde{x}^{i}$ is attainable for $i$. Therefore, it holds that $\bar{u}^{i}(\tilde{y}, \tilde{t}) \geq u^{i}\left(\tilde{x}^{i}\right) \geq \tilde{v}^{i}$ for all $i \in \mathcal{I}$, where the last inequality follows from the concavity of $u^{i}$. Since $V$ is comprehensive from below, we have $\tilde{v} \in V$. Since $v \geq v^{0}$ and $\bar{v} \geq v^{0}$, it follows that $\tilde{v} \in V^{+}$.

The normal of a convex subset $C$ of $\mathbb{R}^{m}$ at a point $\bar{c}$ in $C$ is defined as the set of vectors $c^{*} \in \mathbb{R}^{m}$ satisfying $\left\|c^{*}\right\|=1$ and $(c-\bar{c}) \cdot c^{*} \leq 0$ for every $c \in C$. Equivalent to the uniqueness of the normal at every point in the boundary $\partial C$, we may assume that $\partial C$ is a $C^{1}$ manifold; see Rockafellar (1970).

Lemma 3.5 At any point of $\partial V^{+}$there is a unique vector in the normal to $V$.

Proof: Take any $\bar{v} \in \partial V^{+}$. Let $(\bar{y}, \bar{t})$ be such that $\bar{v}=\bar{u}(\bar{y}, \bar{t})$ and define $\bar{x}^{i}=\xi^{i}(\bar{y}, \bar{t})$, $i \in \mathcal{I}$.

We define the set $T^{\prime}$ by

$$
T^{\prime}=\left\{\left(\tau^{1}, \ldots, \tau^{I-1}, \tau^{I}\right) \in \mathbb{R}^{I-1} \times \mathbb{R}_{+} \mid \bar{x}_{0}^{I}-\sum_{i \in \mathcal{I}} \tau^{i}>0 ; \forall i \in \mathcal{I} \backslash\{I\}, \bar{x}_{0}^{i}+\tau^{i}>0\right\}
$$

The interpretation of $\tau \in T^{\prime}$ is that owner $i=1, \ldots, I-1$ receives a transfer $\tau^{i}$ in period 0 additional to $\bar{x}_{0}^{i}$, whereas owner $I$ receives an additional transfer $-\sum_{i \in \mathcal{I}} \tau^{i}$. Notice that $\tau^{I}$ corresponds to an amount of resource that is wasted. We define the function $f: T^{\prime} \rightarrow \mathbb{R}^{(S+1) I}$ by $f_{0}^{I}(\tau)=\bar{x}_{0}^{I}-\sum_{i \in \mathcal{I}} \tau^{i}, f_{0}^{i}(\tau)=\bar{x}_{0}^{i}+\tau^{i}, i \in \mathcal{I} \backslash\{I\}$, and $f_{s}^{i}(\tau)=\bar{x}_{s}^{i}, i \in \mathcal{I}$, $s \in \mathcal{S} \backslash\{0\}$. We define $K(\bar{v})$ as the image of $T^{\prime}$ under the function $\phi=\left(u^{1} \circ f^{1}, \ldots, u^{I} \circ f^{I}\right)$. Then $\phi^{-1}$ serves as a $C^{2}$ coordinate system for $K(\bar{v})$ around $\bar{v}$, i.e. $\phi^{-1}$ is injective and surjective, $\phi$ is twice differentiable, and we can show that $\phi^{-1}$ is twice differentiable by means of the inverse function theorem using the property that for all $i \in \mathcal{I}, \partial_{x_{0}^{i}} u^{i}\left(\bar{x}^{i}\right)>0$. It follows that $K(\bar{v})$ is a $C^{2}$ manifold with boundary. Since $K(\bar{v})$ is convex, it has a unique outward normal vector at $\bar{v}$, say $v^{*}$.

We want to show that $v^{*}$ is also the unique normal to $V$ at $\bar{v}$. Suppose to the contrary that there is a normal vector $v^{\prime} \neq v^{*}$ to $V$ at $\bar{v}$. Since $v^{\prime}$ cannot be normal to $K(\bar{v})$, there 
is $v \in K(\bar{v})$ such that $(v-\bar{v}) \cdot v^{\prime}>0$. But $K(\bar{v}) \subset V$, so that $v \in V$ as well, contradicting that $v^{\prime}$ is normal to $V$ at $\bar{v}$.

It may be interesting to note that the proof of differentiability of $\partial V$ does not rely on the differentiability of $\partial Y$, though we have assumed the latter for later purposes.

We have established that the set $V^{+}$is compact and convex, that the set $V$ is comprehensive from below, that all points in $\partial V^{+}$are strongly Pareto-efficient, and there is a unique vector in the normal to $V$ at any point in $\partial V^{+}$. In the non-cooperative bargaining literature, one considers abstract sets of feasible payoffs which are assumed to have these properties. Therefore, our preceding analysis of the set $V^{+}$demonstrates that the model we study in this paper lends itself to the application of results already established in the bargaining literature. In particular, given an $I$-player bargaining protocol of the type which we have in our model, and a set of feasible payoffs with the aforementioned properties, a characterization of subgame-perfect equilibria in stationary strategies is known in the literature. All such equilibria lead to the selection of payoffs in $\partial V^{+}$and are characterized by absence of delay in reaching an agreement. Moreover, it is known that in the limit as $\delta$ goes to one, the payoffs implied by such equilibria converge to a weighted Nash Bargaining Solution, where the weights are given by the vector $\mu$ of recognition probabilities, see Hart and Mas-Colell (1996), Miyakawa (2008), and Laruelle and Valenciano (2007). Britz, Herings, and Predtetchinski (2010) have shown that the result can be suitably generalized to the case where proposers are chosen according to an irreducible Markov process. In this case, the weights of the Nash Bargaining Solution are given by the stationary distribution of this Markov process.

Below, we first give a definition of the weighted Nash Bargaining Solution, and then state the aforementioned convergence result formally as Theorem 3.7.

Definition 3.6 The $\mu$-weighted Nash Bargaining Solution $(\mu-N B S)$ is the payoff allocation $v^{*} \in V^{+}$which solves

$$
\max _{v \in V^{+}} \prod_{i=1}^{I}\left(v^{i}-v^{0 i}\right)^{\mu^{i}}
$$

The $\mu$-weighted Nash Bargaining Solution can be interpreted as the choice of a social planner who has a Cobb-Douglas social welfare function with weights $\mu$ and set of feasible alternatives given by $V^{+}$. The convexity of $V^{+}$as demonstrated in Lemma 3.4 implies that the $\mu$-weighted Nash bargaining solution is unique.

Theorem 3.7 In the limit as $\delta \uparrow 1$, the payoffs of all subgame perfect bargaining equilibria in stationary strategies converge to the $\mu-N B S$. 
We finally argue that it is irrelevant whether negotiations are on the implied payoffs directly or on a production plan and transfers since they are in a one to one relationship between each other.

Theorem 3.8 For every $\bar{v} \in \partial V^{+}$there is a unique $(\bar{y}, \bar{t}) \in B$ such that $\bar{v}=\bar{u}(\bar{y}, \bar{t})$.

Proof: Consider a payoff vector $\bar{v} \in \partial V^{+}$and $(y, t),(\bar{y}, \bar{t}) \in B$ such that $\bar{u}(y, t)=$ $\bar{u}(\bar{y}, \bar{t})=\bar{v}$. We want to show that $(y, t)=(\bar{y}, \bar{t})$. We have that $\xi^{i}(y, t) \gg 0$ and $\xi^{i}(\bar{y}, \bar{t}) \gg 0$ for all $i \in \mathcal{I}$.

Suppose that there exists some $i^{\prime} \in \mathcal{I}$ such that $\xi^{i^{\prime}}(y, t) \neq \xi^{i^{\prime}}(\bar{y}, \bar{t})$. Since utility functions are strictly concave on $\mathbb{R}_{++}^{S+1}$ by Assumption 2.2.3, it holds that $\bar{u}^{i^{\prime}}(\tilde{y}, \tilde{t}) \geq u^{i^{\prime}}\left(\tilde{x}^{i^{\prime}}\right)>\bar{v}^{i^{\prime}}$, where $\tilde{x}^{i^{\prime}}=\alpha \xi^{i^{\prime}}(y, t)+(1-\alpha) \xi^{i^{\prime}}(\bar{y}, \bar{t})$ and $(\tilde{y}, \tilde{t})=\alpha(y, t)+(1-\alpha)(\bar{y}, \bar{t})$ for some $\alpha \in(0,1)$. But then $\bar{u}(\tilde{y}, \tilde{t}) \geq \bar{v}$ with strict inequality in component $i^{\prime}$. Thus, we have found an element of $V^{+}$which Pareto-dominates $\bar{v}$, a contradiction. We have shown that $\xi(y, t)=\xi(\bar{y}, \bar{t})$.

Now suppose that $y \neq \bar{y}$ and define $(\tilde{y}, \tilde{t})$ as before. By strict convexity of $Y$, there is $y^{\prime} \in Y$ such that $y^{\prime} \gg \tilde{y}$, and it follows that $\bar{u}\left(y^{\prime}, \tilde{t}\right) \gg \bar{v}$, a contradiction to $\bar{v} \in \partial V^{+}$. We have shown that $y=\bar{y}$.

For all $i \in \mathcal{I}$, we know that $\bar{u}^{i}$ is strictly increasing in the transfer, given the production plan. It follows that $t=\bar{t}$.

Theorem 3.7 characterizes the equilibrium payoffs of the bargaining procedure. Now, we will analyze the production and transfer decisions which lead to these payoffs.

Definition 3.9 The tuple $(x, y, t, z) \in \mathbb{R}_{++}^{(S+1) I} \times B \times \mathbb{R}^{J I}$ is a bargaining equilibrium if $\bar{u}(y, t)=v^{*}$, where $v^{*} \in V^{+}$is the $\mu$-weighted Nash Bargaining Solution, and $(x, z)$ are optimal consumption bundles and asset portfolios given $(y, t)$.

The convexity of $V^{+}$implies that the $\mu$-NBS $v^{*}$ is unique. We have shown in Theorem 3.8 that any efficient and individually rational payoff allocation, and thus the $\mu^{-}$ weighted NBS, is supported by a unique $(y, t) \in B$, and indeed by a unique $(x, y, t, z) \in$ $\mathbb{R}_{++}^{(S+1) I} \times B \times \mathbb{R}^{J I}$, since optimal consumption plans and portfolio choices were argued to be unique for $(y, t) \in B$. We have therefore obtained the following result.

Theorem 3.10 The bargaining equilibrium is unique. 


\section{Weighted Nash Bargaining Solution}

To characterize the bargaining equilibrium, we introduce a twice differentiable quasi-convex transformation function $f: \mathbb{R}^{S+1} \rightarrow \mathbb{R}$ to describe the production possibility set. We choose $f$ such that $Y=\left\{y \in \mathbb{R}^{S+1} \mid f(y) \leq 0\right\}$. If $y \in \partial Y$, the gradient of $f(y)$ corresponds to the outward normal vector to $Y$ at $y$. It holds that $\partial f(y) \gg 0$ and $\alpha^{\top} \partial^{2} f(y) \alpha>0$ for all $\alpha \in \mathbb{R}^{S+1} \backslash\{0\}$ such that $\partial f(y) \alpha=0$.

It may be the case that the set $V^{+}$contains only the point $v^{0}$. Then, the $\mu$-weighted Nash product is equal to $v^{0}$. It follows immediately from Theorem 3.8 that $y=0$ and $t=0$, but owners might still be active on the asset markets. In this case owners have nothing to gain from production by the firm, which renders the bargaining problem uninteresting. At a later stage, we will show formally that this uninteresting case can only occur degenerately.

In what follows, we will focus on the case in which $V^{+}$contains points different from $v^{0}$. In that case, Lemma 3.2 implies that the set $V^{++}=\left\{v \in V^{+} \mid v \gg v^{0}\right\}$ of strictly individually rational elements of $V$ is non-empty. Since all the weights $\mu$ are strictly positive, it follows that the $\mu-\mathrm{NBS} v^{*}$ strictly exceeds $v^{0}$ in each component. Moreover, the set $\partial V^{++}=\partial V \cap V^{++}$of strictly individually rational and Pareto-efficient payoff allocations is parameterized by all the $\mu$-Nash Bargaining Solutions when we vary $\mu$. We proceed to derive the first-order conditions for maximizing the $\mu$-weighted Nash product.

Let $(x, y, t, z)$ be a bargaining equilibrium. It is immediate that the feasibility constraints $f(y) \leq 0$ and $\sum_{i \in \mathcal{I}} t^{i} \leq 0$ hold with equality. The optimization problem involved leads to the following first-order conditions:

$$
\begin{aligned}
\sum_{i \in \mathcal{I}} \frac{\theta^{i} \mu^{i}}{u^{i}\left(x^{i}\right)-v^{0 i}} \partial_{x_{s}^{i}} u^{i}\left(x^{i}\right) & =\lambda \partial_{y_{s}} f(y), s \in \mathcal{S}, \\
\frac{\mu^{i}}{u^{i}\left(x^{i}\right)-v^{0 i}} \partial_{x_{0}^{i}} u^{i}\left(x^{i}\right) & =\nu, i \in \mathcal{I}, \\
\left(\partial_{x_{0}^{i}} u^{i}\left(x^{i}\right), \partial_{x_{1}^{i}} u^{i}\left(x^{i}\right), \ldots, \partial_{x_{S}^{i}} u^{i}\left(x^{i}\right)\right) W & =0, i \in \mathcal{I}, \\
x^{i} & =\omega^{i}+\theta^{i} y+e(0) t^{i}+W z^{i}, i \in \mathcal{I}, \\
f(y) & =0, \\
\sum_{i \in \mathcal{I}} t^{i} & =0,
\end{aligned}
$$

where $\lambda$ and $\nu$ are Lagrangian parameters corresponding to the equations specifying the feasibility of the production plan and the transfer scheme, respectively. Equation (3) corresponds to the standard conditions for optimal portfolio choice. We remark that the first-order conditions are both necessary and sufficient. 
We denote the $S$-dimensional vector of marginal rates of substitution by $\nabla u^{i}\left(x^{i}\right)$, so $\nabla_{s} u^{i}\left(x^{i}\right)=\partial_{x_{s}^{i}} u^{i}\left(x^{i}\right) / \partial_{x_{0}^{i}} u^{i}\left(x^{i}\right)$ for $s=1, \ldots, S$. Similarly, we denote the $S$-dimensional vector of marginal rates of transformation by $\nabla f(y)$, so $\nabla_{s} f(y)=\partial_{y_{s}} f(y) / \partial_{y_{0}} f(y)$ for $s=1, \ldots, S$. When we substitute $\mu^{i} /\left(u^{i}\left(x^{i}\right)-v^{0 i}\right)=\nu / \partial_{x_{0}^{i}} u^{i}\left(x^{i}\right)$ in the system of equations (1), we find

$$
\begin{aligned}
\nu & =\lambda \partial_{y_{0}} f(y), \\
\sum_{i \in \mathcal{I}} \theta^{i} \nabla u^{i}\left(x^{i}\right) & =\nabla f(y) .
\end{aligned}
$$

The last equality implies that the marginal rates of transformation vector is equal to the $\theta$-weighted average of the owners' marginal rates of substitution vectors. Notice that due to potential market incompleteness, it is not guaranteed that marginal rates of substitution vectors are all equal or are equal to the marginal rate of transformation vector. Since the marginal rate of transformation vector corresponds to the outward normal vector to $Y$ at $y$, we find that $(1, \nabla f(y)) y \geq(1, \nabla f(y)) y^{\prime}$ for all $y^{\prime} \in Y$, and therefore it holds that the production plan chosen in a bargaining equilibrium maximizes

$$
y_{0}+\left(\sum_{i \in \mathcal{I}} \theta^{i} \nabla u^{i}\left(x^{i}\right)\right) y_{-0}
$$

over all production plans in $Y$. The corresponding objective function of the firm is known as the Drèze criterion. We have thereby shown the following result.

Theorem 4.1 In a bargaining equilibrium, the production plan is chosen according to the Drèze criterion.

For $i \in \mathcal{I}$, for $x^{i}$ with $u^{i}\left(x^{i}\right)>v^{0 i}$, define $\eta^{i}\left(x^{i}\right)=\partial_{x_{0}^{i}} u^{i}\left(x^{i}\right) /\left(u^{i}\left(x^{i}\right)-v^{0 i}\right)$. Then, (2) is equivalent to

$$
\mu^{i} \eta^{i}\left(x^{i}\right)=\nu, \quad i \in \mathcal{I}
$$

In Aumann and Kurz (1977), $\eta^{i}\left(x^{i}\right)$ is considered a measure of the owner's boldness. Consider a gamble where an owner $i$ receives utility $v^{0 i}$ with probability $p$ and $u^{i}\left(x^{i}+\varepsilon e(0)\right)$ with probability $1-p$, where $\varepsilon>0$. Let $p^{i}\left(x^{i}, \varepsilon\right)$ be the maximum probability for which owner $i$ weakly prefers the gamble over consuming $x^{i}$ for sure. As pointed out in Roth (1989), boldness corresponds to the maximum probability for which owner $i$ is willing to accept the gamble, per dollar of additional gains, when $\varepsilon$ tends to zero. That is, $\eta^{i}\left(x^{i}\right)=\lim _{\varepsilon \downarrow 0} p^{i}\left(x^{i}, \varepsilon\right) / \varepsilon$. Aumann and Kurz (1977) identify the point where boldness 
is equal across all owners as the Nash Bargaining Solution. The above condition says that a weighted Nash Bargaining Solution is the point where the product of boldness and probability to propose is equal for all owners. In the special case where all owners have the same probability to propose, it follows that at a bargaining equilibrium, all owners have equal boldness. Although Aumann and Kurz (1977) define boldness in a context with a single good, here we obtain a similar specification since only the marginal utility of consumption in state 0 enters $\eta^{i}\left(x^{i}\right)$.

By definition of $\nabla u^{i}\left(x^{i}\right)$, we can write Equation (3) as

$$
\nabla u^{i}\left(x^{i}\right) A=q, \quad i \in \mathcal{I} .
$$

These are simply the conditions for optimal portfolio choice for each owner. When markets are complete, for instance when $A$ is the identity, marginal rate of substitution vectors are all equal to each other and to the marginal rate of transformation vector.

The next result specifies the first-order conditions for constrained Pareto optimality and relates them to the ones corresponding to the bargaining equilibrium.

Theorem 4.2 It holds that $\bar{u}(y, t) \in \partial V$ if and only if $(x, y, t, z) \in \mathbb{R}_{++}^{(S+1) I} \times B \times \mathbb{R}^{J I}$ satisfies

$$
\begin{array}{rlr}
x^{i} & =\omega^{i}+\theta^{i} y+e(0) t^{i}+W z^{i}, i \in \mathcal{I} \\
\sum_{i \in \mathcal{I}} \theta^{i} \nabla u^{i}\left(x^{i}\right) & =\nabla f(y), & \\
\nabla u^{i}\left(x^{i}\right) A & =q, & \\
f(y) & =0, & \\
\sum_{i \in \mathcal{I}} t^{i} & =0 . &
\end{array}
$$

Moreover, $(x, y, t, z)$ is a bargaining equilibrium with $\bar{u}(y, t) \in V^{++}$if and only if it satisfies Equations (7)-(11), and

$$
\mu^{i} \eta^{i}\left(x^{i}\right)-\mu^{I} \eta^{I}\left(x^{i}\right)=0, \quad i \in \mathcal{I} \backslash\{I\}
$$

Proof: Equations (7)-(11) follow from the same derivations as in this section when applied to the maximization of the function $\prod_{i=1}^{I}\left(v^{i}\right)^{\mu^{i}}$ for arbitrarily chosen strictly positive weights $\mu^{i}$ and correspond to standard constrained Pareto optimality conditions. For a bargaining equilibrium with utilities in $V^{++}$, the equations follow from the derivations in the beginning of this section. 
The requirement $\bar{u}(y, t) \in V^{++}$is added to rule out the less interesting case where the bargaining equilibrium satisfies $\bar{u}(y, t)=v^{0}$. In that case Equations (7)-(11) are still valid, but $\eta^{i}\left(x^{i}\right)$ is not well-defined since $u^{i}\left(x^{i}\right)$ is equal to $v^{0 i}$.

We have shown that in the limit of subgame perfect equilibria in stationary strategies, the owners' bargaining procedure leads to payoffs corresponding to the $\mu$-weighted Nash Bargaining Solution. Moreover, in the previous theorem, we have translated this result on the equilibrium payoffs into a result on the production plan and transfer scheme chosen by the bargaining procedure. We will now give a formal definition of a competitive equilibrium and begin contrasting it with our findings.

Definition 4.3 The tuple $(x, y, t, z) \in \mathbb{R}_{++}^{(S+1) I} \times B \times \mathbb{R}^{J I}$ is a competitive equilibrium if

$$
\begin{array}{rlr}
x^{i} & =\omega^{i}+\theta^{i} y+e(0) t^{i}+W z^{i}, & i \in \mathcal{I} \\
\sum_{i \in \mathcal{I}} \theta^{i} \nabla u^{i}\left(x^{i}\right) & =\nabla f(y), & \\
\nabla u^{i}\left(x^{i}\right) A & =q, & i \in \mathcal{I}, \\
f(y) & =0, & \\
t & =0 . &
\end{array}
$$

The definition of competitive equilibrium corresponds to what Magill and Quinzii (1996) define as a stock market equilibrium, when taking into account that shares are not traded. Because of this latter property, the name stock market equilibrium would be inappropriate, and we have chosen the name competitive equilibrium. We see that both the bargaining equilibrium and the competitive equilibrium require constrained Pareto-optimality, optimal choice of portfolios by all owners, and efficient production. Moreover, both notions are consistent with the Drèze criterion. The difference between both approaches is the selection made from the outcomes which satisfy constrained Pareto optimality, and which would be called pseudo equilibria or Lindahl equilibria in Drèze (1974). We next present a formal definition of such equilibria, and call them Drèze equilibria.

Definition 4.4 The tuple $(x, y, t, z) \in \mathbb{R}_{++}^{(S+1) I} \times B \times \mathbb{R}^{J I}$ is a Drèze equilibrium if

$$
\begin{array}{rlrl}
x^{i} & =\omega^{i}+\theta^{i} y+e(0) t^{i}+W z^{i}, & & i \in \mathcal{I}, \\
\sum_{i \in \mathcal{I}} \theta^{i} \nabla u^{i}\left(x^{i}\right) & =\nabla f(y), & \\
\nabla u^{i}\left(x^{i}\right) A & =q, & i \in \mathcal{I}, \\
f(y) & =0, & \\
\sum_{i \in \mathcal{I}} t^{i} & =0 . &
\end{array}
$$


The competitive equilibrium chooses a Drèze equilibrium allocation which does not require transfers. Bargaining power or the disagreement point play no role in this selection. Under the bargaining equilibrium, one chooses the unique allocation which can be reached by non-wasteful transfers and at which the $\mu$-weighted boldness of all owners is equal. Both the bargaining equilibrium and the competitive equilibrium are Drèze equilibria.

\section{Producer Choice}

We have studied the equilibrium production and transfer decision of the firm resulting from the bargaining procedure. In the last section, we have characterized the outcome of the bargaining procedure and given a first comparison to the competitive equilibrium. In this section, we make use of the characterization given in Theorem 4.2 in order to study the bargaining equilibrium in much more detail and explore its relation to important concepts well-established in the literature, such as value-maximization and the competitive equilibrium.

Any matrix of security payoffs $W$ implies a set $\Pi$ of (normalized) state prices. A production plan is said to be value-maximizing if it is optimal with regard to some element of $\Pi$ (DeMarzo 1993). It turns out that the set of value-maximizing production plans is closely related to the strictly individually rational Pareto-boundary of $V$, parameterized by the weights $\mu$ of the Nash Bargaining Solution, which are in turn given by the recognition probabilities of the bargaining procedure.

Definition 5.1 A production plan $\bar{y} \in Y$ is value-maximizing if there is a state price vector $\pi \in \Pi$ such that for all $y \in Y$,

$\pi y \leq \pi \bar{y}$

In what follows, we show how our previous characterization of the $\mu$-weighted NBS relates to the value-maximization concept:

Lemma 5.2 A production plan $\bar{y} \in Y$ is value-maximizing if and only if it satisfies

$$
\begin{aligned}
\nabla f(\bar{y}) A & =q, \\
f(\bar{y}) & =0 .
\end{aligned}
$$

Proof: This follows from the standard first-order conditions.

Theorem 4.2 and Lemma 5.2 imply that $\partial V$ is supported only by production plans which are value-maximizing. In the special case with complete markets, $\Pi$ is single-valued and value-maximization reduces to the usual profit-maximization. 
Corollary 5.3 If $S=J$, then every $v \in \partial V$ is supported by the profit-maximizing production plan.

If markets are complete, the production decision of the firm in a bargaining equilibrium is thus consistent with the usual profit-maximizing predictions of competitive equilibrium. However, the distribution of bargaining power will determine how the firm's profits are to be divided among its owners. The bargaining equilibrium utilities may therefore differ from the competitive equilibrium utilities even if markets are complete.

For the remainder of the section, we use the notation

$$
\begin{aligned}
u_{s s^{\prime}}^{i} & =\partial^{2} u^{i}\left(x^{i}\right) / \partial x_{s}^{i} \partial x_{s^{\prime}}^{i}, \\
\nabla_{s} u^{i}\left(x^{i}\right) & =\left(\partial u^{i}\left(x^{i}\right) / \partial x_{s}^{i}\right) /\left(\partial u^{i}\left(x^{i}\right) / \partial x_{0}^{i}\right), s \in \mathcal{S} \backslash\{0\}, \\
\hat{u}_{s s^{\prime}}^{i} & =\partial\left(\nabla_{s} u^{i}\left(x^{i}\right)\right) / \partial x_{s^{\prime}}^{i} .
\end{aligned}
$$

We summarize the second-order derivatives in matrices

$$
U^{i}=\left(\begin{array}{cccc}
u_{00}^{i} & u_{01}^{i} & \cdots & u_{0 S}^{i} \\
u_{10}^{i} & u_{11}^{i} & \cdots & u_{1 S}^{i} \\
\vdots & \vdots & \ddots & \vdots \\
u_{S 0}^{i} & u_{S 1}^{i} & \cdots & u_{S S}^{i}
\end{array}\right) \text { and } \hat{U}^{i}=\left(\begin{array}{cccc}
\hat{u}_{10}^{i} & \hat{u}_{11}^{i} & \cdots & \hat{u}_{1 S}^{i} \\
\vdots & \vdots & \ddots & \vdots \\
\hat{u}_{S 0}^{i} & \hat{u}_{S 1}^{i} & \cdots & \hat{u}_{S S}^{i}
\end{array}\right) .
$$

Similarly, we write

$$
\begin{aligned}
f_{s s^{\prime}} & =\partial^{2} f(y) / \partial y_{s} \partial y_{s^{\prime}}, \\
\nabla_{s} f(y) & =\left(\partial f(y) / \partial y_{s}\right) /\left(\partial f(y) / \partial y_{0}\right), s \in \mathcal{S} \backslash\{0\}, \\
\hat{f}_{s s^{\prime}} & =\partial\left(\nabla_{s} f(y)\right) / \partial y_{s^{\prime}} .
\end{aligned}
$$

and use the matrices

$$
F=\left(\begin{array}{cccc}
f_{00} & f_{01} & \cdots & f_{0 S} \\
f_{10} & f_{11} & \cdots & f_{1 S} \\
\vdots & \vdots & \ddots & \vdots \\
f_{S 0} & f_{S 1} & \cdots & f_{S S}
\end{array}\right) \text { and } \hat{F}=\left(\begin{array}{cccc}
\hat{f}_{10} & \hat{f}_{11} & \cdots & \hat{f}_{1 S} \\
\vdots & \vdots & \ddots & \vdots \\
\hat{f}_{S 0} & \hat{f}_{S 1} & \cdots & \hat{f}_{S S}
\end{array}\right)
$$

Theorem 5.4 The set of value-maximizing production plans is an $(S-J)$-dimensional manifold. 
Proof: We have to show that the matrix of partial derivatives of the following system of equations

$$
\begin{aligned}
\nabla f(y) A-q & =0 \\
f(y) & =0
\end{aligned}
$$

has row rank $J+1$ when evaluated at a solution $y$, after which the result follows from counting equations and unknowns. To this end, we have to show that the rows of the matrix

$$
\left(\begin{array}{c}
A^{\top} \hat{F} \\
\partial f(y)
\end{array}\right)
$$

are linearly independent. We first rewrite the entry in row $j$ and column $s^{\prime}$ of $A^{\top} \hat{F}$ as follows:

$$
\begin{aligned}
{\left[A^{\top} \hat{F}\right]_{j}^{s^{\prime}} } & =\sum_{s=1}^{S} A_{s}^{j} \hat{f}_{s s^{\prime}} \\
& =\sum_{s=1}^{S} A_{s}^{j}\left(\frac{f_{s s^{\prime}} f_{0}-f_{s} f_{0 s^{\prime}}}{\left(f_{0}\right)^{2}}\right) \\
& =\frac{1}{f_{0}}\left[\sum_{s=1}^{S} A_{s}^{j} f_{s s^{\prime}}-\sum_{s=1}^{S} A_{s}^{j} \nabla_{s} f f_{0 s^{\prime}}\right] \\
& =\frac{1}{f_{0}}\left[\sum_{s=1}^{S} A_{s}^{j} f_{s s^{\prime}}-q_{j} f_{0 s^{\prime}}\right] \\
& =\left[\frac{1}{f_{0}} W^{\top} F\right]_{j}^{s^{\prime}} .
\end{aligned}
$$

The second line follows by applying the quotient rule, and the fourth line results from $\nabla f(y) A=q$. Hence, we have that $A^{\top} \hat{F}=\left(1 / f_{0}\right) W^{\top} F$. Now suppose by way of contradiction that there is a row vector $\alpha \in \mathbb{R}^{J}$ and $\beta \in \mathbb{R}$ with $(\alpha, \beta) \neq 0$ such that

$$
\alpha A^{\top} \hat{F}+\beta \partial f(y)=0 .
$$

From right-multiplication by $W$ and $\partial f(y) W=0$, we obtain $\alpha A^{\top} \hat{F} W=0$. From rightmultiplication by $\alpha^{\top}$ and substitution of the previously derived expression for $A^{\top} \hat{F}$, we find that $\alpha W^{\top} F W \alpha^{\top}=0$. Since $f$ is differentiably quasi-convex, the last equation implies $\partial f(y) W \alpha^{\top} \neq 0$, contradicting the fact that $\partial f(y) W=0$. 
We have assumed that $U^{i}$ is negative definite, implying that it is of full rank. In order to prove the next theorem, an important auxiliary result is that also the matrix of normalized second-order derivatives $\hat{U}^{i}$ has linearly independent rows. This is shown in the following lemma.

Lemma 5.5 The matrix $\hat{U}^{i}$ has linearly independent rows.

Proof: Suppose by way of contradiction that there is a row vector $\alpha \in \mathbb{R}^{S} \backslash\{0\}$ such that $\alpha \hat{U}^{i}=0$. By definition of $\hat{U}^{i}$ and the quotient rule

$$
\sum_{s=1}^{S} \alpha_{s}\left[\frac{u_{s s^{\prime}}^{i}}{u_{0}^{i}}-\frac{u_{s}^{i} u_{0 s^{\prime}}^{i}}{\left(u_{0}^{i}\right)^{2}}\right]=0, \quad s^{\prime}=0, \ldots, S,
$$

so we have that

$$
\sum_{s=1}^{S} \alpha_{s} u_{s s^{\prime}}^{i}-\sum_{s=1}^{S} \alpha_{s} \frac{u_{s}^{i}}{u_{0}^{i}} u_{0 s^{\prime}}^{i}=0, \quad s^{\prime}=0, \ldots, S .
$$

Now define a vector $\alpha^{\prime}=\left(-\sum_{s=1}^{S} \alpha_{s} \frac{u_{s}^{i}}{u_{0}^{i}}, \alpha_{1}, \ldots, \alpha_{S}\right)$. Then, $\sum_{s=0}^{S} \alpha_{s}^{\prime} u_{s s^{\prime}}^{i}=0$, contradicting the assumption that $U^{i}$ is negative definite.

Consider a particular $v \in \partial V$. If there exists a production plan $y \in Y$ and asset portfolios $z^{1}, \ldots, z^{I}$ such that $u^{i}\left(\omega^{i}+\theta^{i} y+W z^{i}\right)=v^{i}$ for all $i \in \mathcal{I}$, then we say that the point $v$ is supported without transfers. Notice that competitive equilibria lead to points in $\partial V$ which are supported without transfers.

In what follows, we will parameterize the economy by the initial endowments $\omega$ and the bargaining weights $\mu$. From now on, we will make this explicit by using the notation $\partial V_{\omega}$.

We state a number of results which all rely on a similar proof strategy. In each case, we phrase the problem of interest in such a way that it amounts to finding the dimension of the solution set of some system of equations. Each equation in this system can be identified with the zero of a function in which the endowment schedule $\omega$ and/or the bargaining weights $\mu$ are parameters, while the variables are production plans, transfer schemes, and asset portfolios. We summarize the relevant partial derivatives of these functions with respect to the parameters and the variables in a matrix, and prove that this matrix has linearly independent rows. The parametric transversality theorem then implies that the equations are linearly independent for almost all choices of the parameters. The pre-image theorem is then invoked to find the dimension of the solution set.

An important auxiliary result is that, generically in $\omega$, the disagreement point $v_{\omega}^{0}$ lies in the interior of the bargaining set. 
Lemma 5.6 There is an open set $\Omega^{*} \subset \mathbb{R}_{++}^{(S+1) I}$ of full Lebesgue measure such that for all endowments $\omega \in \Omega^{*}$, the payoff allocation $v_{\omega}^{0}$ which arises from the optimal portfolio choice of all owners given no production and no transfers, does not belong to $\partial V_{\omega}^{+}$.

Proof: Consider the following system of equations in $\omega$ and $z$, where $\omega \in \mathbb{R}_{++}^{(S+1) I}$ and $\omega^{i}+W z^{i} \in \mathbb{R}_{++}^{S+1}$ for all $i \in \mathcal{I}$.

$$
\begin{aligned}
\sum_{i \in \mathcal{I}} \theta^{i} \nabla u^{i}\left(\omega^{i}+W z^{i}\right)-\nabla f(0) & =0 \\
\nabla u^{i}\left(\omega^{i}+W z^{i}\right) A-q & =0, i \in \mathcal{I}, \\
\nabla f(0) A-q & =0 .
\end{aligned}
$$

It holds that $v_{\omega}^{0} \in \partial V_{\omega}^{+}$if and only if (12)-(14) has a solution, as follows from the characterization of the boundary in Theorem 4.2 by setting $y=0$ and $t=0$. We observe that condition (14) is independent of $\omega$. If (14) does not hold, then $v_{\omega}^{0} \notin \partial V_{\omega}^{+}$. In particular, by Assumption 2.3.3, condition (14) fails whenever markets are complete. Now consider the case where condition (14) does hold, and hence $S>J$. Consider the derivatives of conditions (12) and (13) above with respect to $\omega^{I}, \omega^{1}, \ldots, \omega^{I-1}$, which can be written in matrix form as

$$
N=\left(\begin{array}{ccccc}
\theta^{I} \hat{U}^{I} & \theta^{1} \hat{U}^{1} & \ldots & \cdots & \theta^{I-1} \hat{U}^{I-1} \\
0 & A^{\top} \hat{U}^{1} & 0 & \cdots & 0 \\
0 & 0 & \ddots & 0 & 0 \\
\vdots & \vdots & 0 & \ddots & 0 \\
0 & 0 & \cdots & 0 & A^{\top} \hat{U}^{I-1}
\end{array}\right)
$$

Lemma 5.5 and the full column rank of $A$ imply that the diagonal blocks $\theta^{I} \hat{U}^{I}$ and $A^{\top} \hat{U}^{i}, i=1, \ldots, I-1$, have linearly independent rows. Therefore, the rows of $N$ are independent. By the parametric transversality theorem, this implies that the derivatives of the aforementioned expressions with respect to $z$ alone are linearly independent for almost all $\omega \in \mathbb{R}_{++}^{(S+1) I}$. Applying the pre-image theorem and counting unknowns and equations, we find that the set of solutions to the above system is a manifold of dimension $J-S<0$ for almost all $\omega$. We have shown that the set $\Omega^{*}$ of endowments $\omega$ such that $v_{\omega}^{0} \notin \partial V_{\omega}^{+}$is of full Lebesgue measure. It holds that $v_{\omega}^{0}$ is a continuous function of $\omega$, and $\partial V_{\omega}^{+}$is an upper-hemi-continuous correspondence in $\omega$. Hence, if $v_{\bar{\omega}}^{0} \notin \partial V_{\bar{\omega}}^{+}$for some particular $\bar{\omega} \in \Omega^{*}$, then $v_{\omega}^{0} \notin \partial V_{\omega}^{+}$for all $\omega$ in a sufficiently small neighborhood of $\bar{\omega}$. Therefore, $\Omega^{*}$ is open, and the lemma follows. 
Theorem 5.7 There is an open set $P^{*} \subset \mathbb{R}_{++}^{(S+1) I} \times \Delta_{I}$ of full Lebesgue measure such that for all $(\omega, \mu) \in P^{*}$, the bargaining equilibrium involves non-zero transfers.

Proof: We will introduce a system of equations in $\omega, \mu, y, z$, and $\bar{z}$, where the variables are restricted to satisfy the following conditions.

$$
\begin{aligned}
\mu^{i} & >0, i \in \mathcal{I}, \\
\omega^{i} & \gg 0, i \in \mathcal{I}, \\
\omega^{i}+\theta^{i} y+W z^{i} & \gg 0, i \in \mathcal{I}, \\
\omega^{i}+W \bar{z}^{i} & \gg 0, i \in \mathcal{I}, \\
u^{i}\left(\omega^{i}+\theta^{i} y+W z^{i}\right)-u^{i}\left(\omega^{i}+W \bar{z}^{i}\right) & >0, i \in \mathcal{I} .
\end{aligned}
$$

The set of $(\omega, \mu, y, z, \bar{z})$ satisfying these restrictions is open, and therefore a manifold. The system of equations is as follows.

$$
\begin{aligned}
\nabla f(y) A-q & =0, \\
f(y) & =0, \\
\nabla u^{i}\left(\omega^{i}+\theta^{i} y+W z^{i}\right) A-q & =0, i \in \mathcal{I} \backslash\{I\}, \\
\sum_{i \in \mathcal{I}} \theta^{i} \nabla u^{i}\left(\omega^{i}+\theta^{i} y+W z^{i}\right)-\nabla f(y) & =0, \\
\gamma^{1}\left(\omega^{1}, \mu^{1}, y, z^{1}, \bar{z}^{1}\right)-\gamma^{I}\left(\omega^{I}, \mu^{I}, y, z^{I}, \bar{z}^{I}\right) & =0, \\
\nabla u^{i}\left(\omega^{i}+W \bar{z}^{i}\right) A-q & =0, i \in \mathcal{I},
\end{aligned}
$$

where we have defined

$$
\gamma^{i}\left(\omega^{i}, \mu^{i}, y, z^{i}, \bar{z}^{i}\right)=\frac{u^{i}\left(\omega^{i}+\theta^{i} y+W z^{i}\right)-u^{i}\left(\omega^{i}+W \bar{z}^{i}\right)}{\mu^{i} \partial_{0} u^{i}\left(\omega^{i}+\theta^{i} y+W z^{i}\right)}, i \in \mathcal{I} .
$$

Given $(\omega, \mu)$, the solution to this system is a tuple $(y, z, \bar{z})$ such that the production plan $y$, the asset portfolios $z$, and zero transfers correspond to a $\mu$-bargaining equilibrium with $v_{\omega}^{0} \in \partial V_{\omega}^{+}$and, moreover, $\bar{z}$ are asset portfolios corresponding to the disagreement point. We want to show that for generically chosen $(\omega, \mu)$, this system is over-determined. This amounts to proving that the derivatives of the above equations are linearly independent.

Consider a block matrix $M$ in which the first row corresponds to the $J+1$ derivatives of equations (15)-(16), the second row to the $I J+S-J+1$ derivatives of equations (17)-(19), and the third row to the $I J$ derivatives of equation (20). Moreover, the first column gives the derivatives with respect to $y$, the second column with respect to $(\omega, \mu)$, and the third 
column with respect to $\bar{z}$. The proof strategy is to show that $M$ is of the lower-triangular form,

$$
M=\left(\begin{array}{ccc}
* & 0 & 0 \\
& * & 0 \\
& & *
\end{array}\right)
$$

where the diagonal blocks have rank $J+1, I J+S-J+1$, and $I J$, respectively.

The proof of Theorem 5.4 shows that the first diagonal block is of rank $J+1$. The corresponding equations do not involve $(\omega, \mu)$ nor $\bar{z}$. This explains the form of the first row of $M$.

Now consider the last diagonal block of $M$. It corresponds to the block-diagonal matrix

$$
M^{33}=\left(\begin{array}{cccc}
A^{\top} \hat{U}^{1} W & 0 & \cdots & 0 \\
0 & \ddots & & \vdots \\
\vdots & & \ddots & 0 \\
0 & \cdots & 0 & A^{\top} \hat{U}^{I} W
\end{array}\right)
$$

Each block of the matrix above is of rank $J$, reflecting the fact that there is a unique optimal asset portfolio for each agent, given the endowments, production decision, and transfers (which are zero in the case at hand).

We now proceed to the second row of $M$. We argue first that the derivatives of equations (17)-(19) with respect to $\bar{z}$ are all zero. This is obvious for equations (17)-(18) which do not involve $\bar{z}$. With regard to equation (19), the derivative of any $\gamma^{i}$ with respect to $\bar{z}^{i}$ is of the form $\kappa \partial u^{i}\left(\omega^{i}+W \bar{z}^{i}\right) W$, where $\kappa$ is a scalar. But at the solution to the equation system above, $\bar{z}^{i}$ is chosen optimally as by equation (20), so that $\partial u^{i}\left(\omega^{i}+W \bar{z}^{i}\right) W=0$, as desired.

Now it remains to show that the block in the center of the matrix $M$ is of full rank. It corresponds to the derivatives of equations (17)-(19) with respect to $(\omega, \mu)$. Omitting the derivatives with respect to $\mu^{i}$ for $i=1, \ldots, I-1$, it can be spelled out as follows.

$$
M^{22}=\left(\begin{array}{cccccc}
A^{\top} \hat{U}^{1} & 0 & \cdots & \cdots & 0 & 0 \\
0 & \ddots & & & \vdots & \vdots \\
\vdots & & \ddots & & 0 & 0 \\
0 & \cdots & \cdots & A^{\top} \hat{U}^{I-1} & 0 & 0 \\
\theta^{1} \hat{U}^{1} & \cdots & \cdots & \cdots & \theta^{I} \hat{U}^{I} & 0 \\
\partial_{\omega^{1}} \gamma^{1} & 0 & \cdots & 0 & \partial_{\omega^{I}} \gamma^{I} & \partial_{\mu^{I}} \gamma^{I}
\end{array}\right)
$$

The linear independence of the first $(I-1) J+S-J$ rows of $M^{22}$ has been shown in the proof of Lemma 5.6. We can write $\partial_{\mu^{I}} \gamma^{I}=-\kappa^{I}\left(\mu^{I}\right)^{(-2)}$, where the factor $\kappa^{I}$ is strictly 
positive by the restriction that $u^{i}\left(\omega^{i}+\theta^{i} y+W z^{i}\right)>u^{i}\left(\omega^{i}+W \bar{z}^{i}\right)$ for all $i \in \mathcal{I}$. Also, we have assumed $\mu \gg 0$, thus $\partial_{\mu^{I}} \gamma^{I}<0$, as required.

We have now shown that the rows of $M$ are linearly independent. By the parametric transversality theorem, we can conclude that the set of $(\omega, \mu) \in \mathbb{R}_{++}^{(S+1) I} \times \Delta_{I}$ for which the derivatives with respect to the remaining variables are linearly independent is of full Lebesgue measure. By the preimage theorem, we may then count equations and unknowns and find that the set of $(\omega, \mu) \in \mathbb{R}_{++}^{(S+1) I} \times \Delta_{I}$ for which the equation system is overdetermined is of full Lebesgue measure. Denote the intersection of that set with $\Omega^{*}$ by $P^{*}$. If $(\omega, \mu) \in P^{*}$, then the bargaining equilibrium involves non-zero transfers. $P^{*}$ is of full Lebesgue measure. Moreover, the (unique) bargaining equilibrium is a continuous function of $(\omega, \mu)$. Therefore, if the property of non-zero transfers holds for some $(\bar{\omega}, \bar{\mu}) \in$ $\mathbb{R}_{++}^{(S+1) I} \times \Delta_{I}$, then it also holds for $(\omega, \mu) \in \mathbb{R}_{++}^{(S+1) I} \times \Delta_{I}$ in a sufficiently small neighborhood of $(\bar{\omega}, \bar{\mu})$. Hence, the set $P^{*}$ is open.

Theorem 5.7 says that generically in endowments and bargaining weights the bargaining procedure will lead to transfers. Since competitive equilibria involve zero transfers, bargaining equilibria are generically distinct from competitive equilibria.

Corollary 5.8 There is an open set $P^{*} \subset \mathbb{R}_{++}^{(S+1) I} \times \Delta_{I}$ of full Lebesgue measure such that for all $(\omega, \mu) \in P^{*}$, the bargaining equilibrium is not a competitive equilibrium.

Since by Theorem 3.8 points in $\partial V^{+}$are supported by uniquely defined production plans and transfer schemes, the bargaining equilibrium utilities and the competitive equilibrium utilities are different for almost all endowments and bargaining weights.

Corollary 5.9 There is an open set $P^{*} \subset \mathbb{R}_{++}^{(S+1) I} \times \Delta_{I}$ of full Lebesgue measure such that for all $(\omega, \mu) \in P^{*}$, the bargaining equilibrium utilities are not equal to competitive equilibrium utilities.

This difference in payoff allocation holds for both complete and incomplete markets. In the case of complete markets, we have previously shown that the profit-maximizing production plan is selected by the bargaining procedure. Thus, with complete markets, any difference in payoff allocation must be due to the transfers. With regard to incomplete markets, however, we will show in the sequel of this section that the difference in payoff allocation is not only the result of transfers, but that the chosen production plan is different as well. 
Theorem 5.10 Suppose that markets are incomplete, that is, $J<S$. Then, there is an open set $P^{* *} \subset \mathbb{R}_{++}^{(S+1) I} \times \Delta_{I}$ of full Lebesgue measure such that for all $(\omega, \mu) \in P^{* *}$, the bargaining equilibrium production plan is not a competitive equilibrium production plan.

Proof: We introduce a system of equations in $(\omega, \mu), y, t, z, \hat{z}$, and $\bar{z}$, where the variables are restricted as follows.

$$
\begin{aligned}
\mu^{i} & >0, i \in \mathcal{I}, \\
\omega^{i} & \gg 0, i \in \mathcal{I}, \\
\omega^{i}+\theta^{i} y+e(0) t^{i}+W z^{i} & \gg 0, i \in \mathcal{I}, \\
\omega^{i}+\theta^{i} y+W \hat{z}^{i} & \gg 0, i \in \mathcal{I}, \\
\omega^{i}+W \bar{z}^{i} & \gg 0, i \in \mathcal{I}, \\
u^{i}\left(\omega^{i}+\theta^{i} y+e(0) t^{i}+W z^{i}\right)-u^{i}\left(\omega^{i}+W \bar{z}^{i}\right) & >0, i \in \mathcal{I} .
\end{aligned}
$$

The set of variables satisfying these restrictions is open, and is therefore a manifold. The system of equations under consideration is as follows.

$$
\begin{aligned}
\sum t_{i \in \mathcal{I}} t^{i} & =0, \\
\nabla f(y) A-q & =0, \\
f(y) & =0, \\
\nabla u^{i}\left(\omega^{i}+\theta^{i} y+e(0) t^{i}+W z^{i}\right) A-q & =0, i \in \mathcal{I} \backslash\{I\}, \\
\sum_{i \in \mathcal{I}} \theta^{i} \nabla u^{i}\left(\omega^{i}+\theta^{i} y+e(0) t^{i}+W z^{i}\right)-\nabla f(y) & =0, \\
\gamma^{i}\left(\omega^{i}, \mu^{i}, y, t^{i}, z^{i}, \bar{z}^{i}\right)-\gamma^{I}\left(\omega^{I}, \mu^{I}, y, t^{I}, z^{I}, \bar{z}^{I}\right) & =0, i \in \mathcal{I} \backslash\{I\}, \\
\nabla u^{i}\left(\omega^{i}+\theta^{i} y+W \hat{z}^{i}\right) A-q & =0, i \in \mathcal{I} \backslash\{I\}, \\
\sum_{i \in \mathcal{I}} \theta^{i} \nabla u^{i}\left(\omega^{i}+\theta^{i} y+W \hat{z}^{i}\right)-\nabla f(y) & =0, \\
A^{\top} \nabla u^{i}\left(\omega^{i}+W \bar{z}^{i}\right)-q & =0, i \in \mathcal{I} .
\end{aligned}
$$

In words, the equations specify that -given $(\omega, \mu)-(y, t, z)$ is the $\mu$-bargaining equilibrium, and $(y, 0, \hat{z})$ is the competitive equilibrium, where for $i \in \mathcal{I}$, we have $x^{i}=$ $\omega^{i}+\theta^{i} y+e(0) t^{i}+W z^{i}$ and $\hat{x}^{i}=\omega^{i}+\theta^{i} y+W \hat{z}^{i}$. We want to show that for generically chosen $(\omega, \mu)$, this system is over-determined whenever markets are complete. This is equivalent to proving that the equations in the system above are all linearly independent. 
Let $N$ be a matrix where the first row corresponds to the derivative of equation (21), the second row corresponds to the $J+1$ derivatives of equations (22)-(23), the third row corresponds to $I J+S-J+I-1$ derivatives of equations (24)-(26), the fourth row to the $I J+S-J$ derivatives of equations (27)-(28) and the fifth row corresponds to the $I J$ derivatives of equation (29). Moreover, the first column refers to derivatives with respect to $t$, the second column with respect to $y$, the third column with respect to $(\omega, \mu)$, the fourth column with respect to $\hat{z}$, and the fifth column with respect to $\bar{z}$.

We will show that $N$ is of the lower triangular form,

$$
N=\left(\begin{array}{ccccc}
* & 0 & 0 & 0 & 0 \\
& * & 0 & 0 & 0 \\
& & * & 0 & 0 \\
& & & * & 0 \\
& & & & *
\end{array}\right),
$$

where the diagonal blocks are of full row rank $1, J+1, I J+S-J+I-1, I J+S-J$, and $I J$, respectively. By the parametric transversality theorem, this means that for generic $(\omega, \mu)$, the matrix of derivatives with respect to the remaining variables $(y, z, \hat{z}, \bar{z}, t)$ has linearly independent rows. Applying the pre-image theorem, this will imply that the set of $(y, z, \hat{z}, \bar{z}, t)$ which solve the above equation system given a generic $(\omega, \mu)$ is a manifold of dimension $J-S$, and corresponds therefore to the empty set when $J<S$.

Equation (21) involves only $t$, which explains the form of the first row of $N$. Equations (22)-(23) involve only $y$. This together with Theorem 5.4 explains that the second row of $N$ is of the form indicated above. The linear independence of the $I J$ rows in the last diagonal block of $N$ follows by the same argument as in the proof of Theorem 5.7. Aside from equation (29), the portfolios $\bar{z}$ are only involved in equation (26). But the derivative of (26) with respect to $\bar{z}$ must be zero at the solution to the equation system, whence the zero entries in the fifth column of $N$ - the argument is as in the proof of Theorem 5.7. Similarly, the portfolios $\hat{z}$ are involved only in equations (27)-(28), explaining the zero entries in the fourth column of $N$.

Consider the third diagonal block of $N$. After appropriate permutations of the rows and columns, it can be spelled out as follows. 


$$
N^{33}=\left(\begin{array}{cccccccccc}
A^{\top} \hat{U}^{1} & 0 & \cdots & \cdots & 0 & 0 & 0 & 0 & 0 & 0 \\
0 & \ddots & & & \vdots & \vdots & \vdots & \vdots & \vdots & \vdots \\
\vdots & & \ddots & & 0 & \vdots & \vdots & \vdots & \vdots & \vdots \\
0 & \cdots & \cdots & A^{\top} \hat{U}^{I-1} & 0 & \vdots & \vdots & \vdots & \vdots & \vdots \\
\theta^{1} \hat{U}^{1} & \cdots & \cdots & \cdots & \theta^{I} \hat{U}^{I} & 0 & 0 & 0 & 0 & 0 \\
\partial_{\omega^{1}} \gamma^{1} & 0 & \cdots & 0 & \partial_{\omega^{I}} \gamma^{I} & \partial_{\mu^{1}} \gamma^{1} & 0 & \cdots & 0 & \partial_{\mu^{I}} \gamma^{I} \\
0 & \ddots & & & \vdots & 0 & \ddots & & \vdots & \vdots \\
\vdots & & \ddots & & \vdots & \vdots & & \ddots & 0 & \vdots \\
0 & \cdots & 0 & \partial_{\omega^{I-1}} \gamma^{I-1} & \partial_{\omega^{I}} \gamma^{I} & 0 & \cdots & 0 & \partial_{\mu^{I-1}} \gamma^{I-1} & \partial_{\mu^{I}} \gamma^{I}
\end{array}\right)
$$

The block in the upper left corner has previously been shown to be of rank $I J+S-J$, see the proof of Lemma 5.6. We have argued in the proof of Theorem 5.7 that the terms of the form $\partial_{\mu^{i}} \gamma^{i}$ are strictly positive, which readily implies the full rank $I-1$ of the block in the lower right corner. Let $P^{* *}$ be the intersection of $\Omega^{*}$ with the set of all $(\omega, \mu) \in \mathbb{R}_{++}^{(S+1) I} \times \Delta_{I}$ for which the matrix under consideration has linearly independent rows. We have shown that $P^{* *}$ is of full Lebesgue measure. The bargaining equilibrium is a continuous function of $(\omega, \mu)$, and the competitive equilibria are an upper-hemi-continuous correspondence of $(\omega, \mu)$. If the property of strictly positive distance between the bargaining and competitive equilibrium production plans holds for some $(\bar{\omega}, \bar{\mu}) \in \mathbb{R}_{++}^{(S+1) I} \times \Delta_{I}$, then it is preserved for $(\omega, \mu) \in \mathbb{R}_{++}^{(S+1) I} \times \Delta_{I}$ in a sufficiently small neighborhood around $(\bar{\omega}, \bar{\mu})$.

We had previously shown that generically in endowments and bargaining weights the payoff allocation resulting from the bargaining equilibrium and that resulting from a competitive equilibrium are different. We have seen that in the case of complete markets, the production plan chosen under both approaches is the same, so that the different payoff allocation is merely a result of redistribution via transfers. The last theorem complements these findings by saying that for the case of incomplete markets, the two approaches almost always lead to different production plans.

\section{Conclusion}

We have introduced a non-cooperative bargaining procedure to resolve the conflict among shareholders of a firm when markets are incomplete. In contrast to many existing models, we obtain a unique prediction for the production plan as well as for the resulting payoff 
allocation. This solution is parameterized by the distribution of bargaining power across the different owners. This bargaining power distribution is independent of the shares of ownership.

An important feature of the model is that transfers are possible in equilibrium. The well-known Drèze criterion rules out a production plan which can be Pareto-improved upon by an alternative plan and transfers. The well-known solution concept of a stock market equilibrium satisfies this criterion but requires that the chosen production plan itself should be implemented without transfers. Our solution concept, called a bargaining equilibrium, satisfies the Drèze criterion but does allow for transfers to be made in equilibrium. Indeed, it turns out that transfers will almost always be used. Furthermore, the bargaining equilibrium is derived from an explicit non-cooperative bargaining model. The outcome of the bargaining procedure proposed in this paper is different from the predictions of standard economic theory. If markets are complete, the production decision of the firm is driven by profit-maximization as in the Arrow-Debreu model. However, the profits are redistributed among the owners of the firm in accordance with their bargaining power, which derives from the ability to make a proposal and from the disagreement payoff. In the case of incomplete markets, the production plan adopted under the bargaining procedure almost always fails to be a competitive equilibrium production plan. Non-zero transfers are almost always made. We have given positive support for the use of the Drèze criterion, though the utility gradients of owners implied by our theory differ from the competitive equilibrium ones.

In our bargaining game we have not considered the option for owners to modify ownership shares and/or to sell the firm to outsiders. An intriguing question for future research is whether allowing such possibilities would give support for the criterion proposed by Bisin, Gottardi and Ruta (2009), which loosely speaking corresponds to the maximal utility gradient in the population rather than a weighted average of the owner's utility gradients as a criterion. 


\section{References}

Aumann, R.J., And M. Kurz (1977), "Power and Taxes," Econometrica, 45, 1137-1161.

Bisin, A., P. Gottardi, and G. Ruta (2009), "Equilibrium Corporate Finance," Working Paper, European University Institute.

Bonnisseau, J.-M., And O. Lachiri (2004), "On the Objective of Firms under Uncertainty with Stock Markets," Journal of Mathematical Economics, 40, 493-513.

Britz, V., P.J.J. Herings, And A. Predtetchinski (2010), "Non-cooperative Support for the Asymmetric Nash Bargaining Solution," Journal of Economic Theory, 145, 1951-1967.

DeMarzo, P. (1993), "Majority Voting and Corporate Control: The Rule of the Dominant Shareholder," Review of Economic Studies, 60, 713-734.

Diamond, P.A. (1967), "The Role of a Stock Market in a General Equilibrium Model with Technological Uncertainty," American Economic Review, 57, 759-776.

Dierker, E., H. Dierker, and B. Grodal (2002), "Nonexistence of Constrained Efficient Equilibria When Markets Are Incomplete," Econometrica 70, 1245-1251.

Drèze, J.H. (1974), "Investment under Private Ownership: Optimality, Equilibrium, and Stability," in J.H. Drèze, ed., Allocation under Uncertainty: Equilibrium and Optimality (New York: Macmillan, 1974) 9.

Drèze, J.H., O. Lachiri, and E. Minelli (2009), "Stock Prices, Anticipations and Investment in General Equilibrium," CORE Discussion Paper, 2009/83, 1-46.

Grossman, S.J., and O. Hart (1979), "A Theory of Competitive Equilibrium in Stock Market Economies," Econometrica, 47, 293-330.

Hart, S. And A. Mas-Colell (1996), "Bargaining and Value," Econometrica, 64, 357-380.

Kelsey, D., And F. Milne (1996), "The Existence of Equilibrium in Incomplete Markets and the Objective Function of the Firm," Journal of Mathematical Economics, 25, 229-245.

Laruelle, A., and F.Valenciano (2007), "Bargaining in Committees as an Extension of Nash's Bargaining Theory," Journal of Economic Theory, 132, 291-305.

Magill, M., And M. Quinzi (1996), Theory of Incomplete Markets, MIT Press, Cambridge, Massachusetts.

Miyakawa, T. (2008), "Noncooperative Foundation of $n$-Person Asymmetric Nash Bargaining Solution," Journal of Economics of Kwansei Gakuin University, 62, 1-18.

Rockafellar, R.T. (1970), Convex Analysis, Princeton University Press, Princeton, New Jersey.

Roth, A.E. (1989), "Risk Aversion and the Relationship between Nash's Solution and Subgame Perfect Equilibrium of Sequential Bargaining," Journal of Risk and Uncertainty, 2, $353-365$. 
Tvede, M., And H. Cres (2005), "Voting in Assemblies of Shareholders and Incomplete Markets," Economic Theory, 26, 887-906. 